\title{
Transmission Schemes for Future Access Networks
}

\author{
R. I. Killey, M. S. Erkılınç, D. Lavery, and P. Bayvel \\ Optical Networks Group, UCL (University College London), Department of Electronic and Electrical Engineering, \\ Roberts Building, Torrington Place, London WC1E 7JE, UK \\ r.killey@ucl.ac.uk
}

\begin{abstract}
We review recent work on low-complexity coherent transceivers for future optical access networks, using single-photodiode heterodyne detection with DSP-based linearization and polarization-independent operation through the use of Alamouti coding.

OCIS codes: (060.4510) Optical communications; (060.1660) Coherent communications; (060.2840) Heterodyne
\end{abstract}

\section{Introduction}

Throughput requirements of access networks are rapidly increasing, driven by data-intensive applications including high-definition, low-latency video-on-demand and the Internet of Things [1]. The ITU-T has recently standardized second generation passive optical network (PON) technology, NG-PON2 [2], which exploits time and wavelength domains in order to offer network throughputs of up to $40 \mathrm{~Gb} / \mathrm{s}$. However, projections of required network capacity for optical access networks suggest that the demand may reach ten times this value by 2025 . Due to their scalability, wavelength division multiplexed (WDM) PONs, in which a wavelength is dedicated for each user, have recently been considered by network operators and service providers [3,4].

Since optical amplification is not used in such networks, which typically have high losses due to the fibers and the optical 1:N splitters at the remote nodes, high sensitivity receivers are needed in both the optical line terminal (OLT) at the Central Office, and in the optical network units (ONUs) at the customers' premises. This can be achieved using coherent detection [5]. For the OLT, a high throughput (hundreds of $\mathrm{Gb} / \mathrm{s}$ ) dual-polarization transceiver (TRx) employing intradyne coherent detection and serving multiple ONUs may be a practical solution, since its cost is shared across multiple network users. However, much simpler TRx solutions will be required for the ONUs, since the cost of each ONU is borne by a single user.

Consequently, much research has been carried out on reduced-complexity coherent TRx technology for the ONU. Design simplifications being investigated include polarization-independent signal detection (avoiding the requirement for polarization rotation/splitting), heterodyne detection (avoiding the need for an optical hybrid and allowing the use of a single analog-to-digital converter) and the use of a single photodetector, in place of balanced detectors.

\section{Reduced-complexity coherent receivers for ONUs}

The complexity of a coherent receiver can be significantly reduced if it can be made polarization-independent, and the need for polarization demultiplexing is avoided. While direct-detection receivers are inherently polarizationindependent, single polarization coherent receivers are not; the state of polarization (SOP) of the received signal is random and varies dynamically during transmission through the fiber. If the received signal SOP is not correctly aligned with that of the local oscillator (LO), a loss of signal results. A number of solutions for this problem have been proposed [6-8]. The use of Alamouti coding [9] in a simple polarization-time block coding of the signal has been proposed and demonstrated to achieve polarization-independent coherent reception [10-12]. Although the coding results in a 50\% redundancy, the ONU receiver can operate without the need for received signal and LO polarization alignment. Alamouti-coded transmission and heterodyne detection, using a 3-dB coupler and balanced photodetectors has been demonstrated in a $10.7 \mathrm{~Gb} / \mathrm{s}$ orthogonal frequency division multiplexed quadrature phase shift keying (OFDM-QPSK) system experiment, with just 58 photons-per-bit being required for error-free operation [13].

The use of heterodyne detection with a single photodiode has been investigated in [14]. This provides a saving of one photodiode, but results in signal-signal beating interference (SSBI), in contrast to the case with balanced detection. Solutions that have been proposed to overcome this include the use of a high LO-to-signal power ratio, so that the wanted signal-LO beat terms dominate over the signal-signal beat terms [14], and the use of digital signal processing (DSP) to mitigate the SSBI, e.g. the Kramers-Kronig scheme [15-18].

A possible future low-complexity ONU TRx design, combining Alamouti coding and single photodiode heterodyne detection, is shown in Fig. 1. A heterodyne receiver, based on a 3-dB coupler and a single photodiode, is used to receive the downstream (DS) $M$-ary quadrature amplitude modulated Alamouti-coded signals. The Alamouti coding at the OLT transmitter is employed to achieve polarization-independent signal reception, while signal-signal 
beat interference mitigation is achieved using the Kramers-Kronig scheme in the ONU Rx DSP. A single dualoutput laser doubles as the downstream LO and the upstream Tx optical source, with real-valued modulation (e.g. pulse amplitude modulation) being carried out for the upstream signal using an integrated electroabsorption modulator. Using these techniques, high sensitivity reception can be achieved with a minimal increase in system complexity compared to the direct detection receivers currently used.

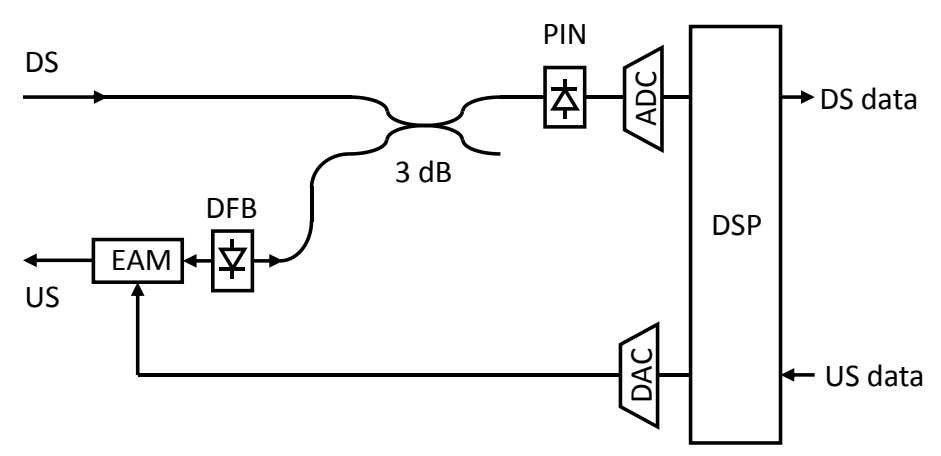

Fig. 1. Optical network unit (ONU) design, using a single photodiode heterodyne receiver, with polarization-independent operation using Alamouti coding for downstream signal detection, and electroabsorption modulator-based transmitter.

This work has been supported by the UK EPSRC UNLOC project.

\section{References}

[1] Huawei Technologies Co. Ltd., Next-generation PON evolution (2013), URL:www.huawei.com/ilink/en/download/HW_077443 (date of access: 08/30/2017).

[2] ITU, ITU-T recommendation G.989.2 Amendment 1 (04/16) 40-Gigabit capable passive optical networks (NG-PON2): General requirements, (2017), URL: https://www.itu.int/rec/T-REC-G.989.2-201604-I!Amd1/en (date of access: 08/08/30/2017).

[3] J.I. Kani, 2010. Enabling technologies for future scalable and flexible WDM-PON and WDM/TDM-PON systems. IEEE Journal of Selected Topics in Quantum Electronics, 16(5), pp.1290-1297.

[4] S. Pachnicke, J. Zhu, M. Lawin, M.H. Eiselt, S. Mayne, B. Quemeneur, D. Sayles, H. Schwuchow, A. Wonfor, P. Marx and M. Fellhofer, 2016. Tunable WDM-PON system with centralized wavelength control. Journal of Lightwave Technology, 34(2), pp.812-818.

[5] D. Lavery, R. Maher, D.S. Millar, B.C. Thomsen, P. Bayvel, and S.J. Savory, 2013. Digital coherent receivers for long-reach optical access networks. Journal of Lightwave Technology, 31(4), pp. 609-620.

[6] I. N. Cano, A. Lerin, V. Polo and J. Prat, 2015. Polarization independent single-PD coherent ONU receiver with centralized scrambling in udWDM-PONs. In European Conference on Optical Communication (ECOC), paper P.7.12.

[7] E. Ciaramella, 2014. Polarization-independent receivers for low-cost coherent OOK systems. IEEE Photon. Technol. Lett., 26(6), pp. 548551.

[8] J. Tabares, V. Polo and J. Prat, 2017. Polarization-independent heterodyne DPSK receiver based on $3 \times 3$ coupler for cost-effective udWDMPON. In Optical Fiber Communication Conference (OFC), paper Th1K.3.

[9] S.M. Alamouti, 1998. A simple transmit diversity technique for wireless communications. IEEE Journal on Selected Areas in Communications, 16(8), pp.1451-1458.

[10] W. Shieh, 2008. Coherent optical OFDM: has its time come? J. Opt. Netw., 7(3), pp. 234-255.

[11] M. S. Erkılınc, D. Lavery, R. Maher, M. Paskov, B.C. Thomsen, R.I. Killey, P. Bayvel, and S.J. Savory. 2015. Polarization-insensitive single balanced photodiode coherent receiver for passive optical networks. In European Conference on Optical Communication (ECOC), paper Th.1.3.3.

[12] M.S. Erkılınc, D. Lavery, B.C. Thomsen, R.I. Killey, P. Bayvel, and S.J. Savory, 2016. Polarization-insensitive single-balanced photodiode coherent receiver for long-reach WDM-PONs. Journal of Lightwave Technology, 34(8), pp. 2034-2041.

[13] M. S. Erkılınc, D. Lavery, B.C. Thomsen, R.I. Killey, S.J. Savory,and P. Bayvel, 2017. Bidirectional wavelength division multiplexed transmission over installed fibre using a simplified optical coherent access transceiver. Nature Communications, 8(1043), 2017.

[14] B. Corcoran, B. Foo, and A.J. Lowery, 2018. Single-photodiode per polarization receiver with signal-signal beat interference suppression through heterodyne detection. Optics Express, 26(3), pp.3075-3086.

[15] A. Mecozzi, C. Antonelli, and M. Shtaif, 2016. Kramers-Kronig coherent receiver. Optica, 3(11), pp.1220-1227.

[16] H. Voelcker, 1966. Demodulation of single-sideband signals via envelope detection. IEEE Transactions on Communication Technology, 14(1), pp.22-30.

[17] Z. Li, M.S. Erkılınç, K. Shi, E. Sillekens, L. Galdino, B.C. Thomsen, P. Bayvel, and R.I. Killey, 2017. SSBI mitigation and the KramersKronig scheme in single-sideband direct-detection transmission with receiver-based electronic dispersion compensation. Journal of Lightwave Technology, 35(10), pp.1887-1893.

[18] X. Chen, C. Antonelli, S. Chandrasekhar, G. Raybon, A. Mecozzi, M. Shtaif, and P. Winzer, 2017. 218-Gb/s single-wavelength, singlepolarization, single-photodiode transmission over $125-\mathrm{km}$ of standard singlemode fiber using Kramers-Kronig detection. In Optical Fiber Communication Conference (paper Th5B-6). Optical Society of America. 